\title{
Dolor en la Artrosis de Rodilla
}

\section{Pain in Knee Osteoarthritis}

\author{
C. Alejandro Álvarez López ${ }^{1}$ Sergio Ricardo Soto-Carrasco ${ }^{20}$ Yenima de la Caridad García Lorenzo ${ }^{3}$ \\ 1 Doctorado en Artroscopia de la Rodilla, Máster en Urgencias Médicas, \\ Especialista en Ortopedia y Traumatología, Profesor Titular, Hospital \\ Address for correspondence Dr. C. Alejandro Álvarez López, MD, PhD, \\ Universitario Manuel Ascunce Domenech, Investigador auxiliar del CITMA, \\ Universidad de Ciencias Médicas, Camagüey, Cuba \\ Universidad de Ciencias Médicas, Calle 2 da No 2. Esq. Lanceros, \\ Reparto La Norma, Camagüey Ciudad. 1, Cuba \\ (e-mail: aal.cmw@infomed.sld.cu).
}

2 Médico cirujano, Asociación Chilena de Seguridad (ACHS), Chillán, Chile

${ }^{3}$ Especialista en Medicina General Integral, Profesor Asistente, Policlínico Universitario Tula Aguilera, Universidad de Ciencias Médicas, Camagüey, Cuba

Rev Chil Ortop Traumatol 2019;60:106-111.

\section{Resumen \\ Palabras clave \\ - artrosis rodilla \\ - dolor rodilla \\ - imagenología artrosis}

El dolor constituye el síntoma fundamental de la artrosis, sus características e interpretación permiten el diagnóstico certero y también conocer la magnitud de esa entidad. El objetivo de este trabajo, es profundizar los conocimientos sobre los elementos más esenciales relacionados con el dolor en la artrosis de la rodilla. Se describen las causas mecánicas y bioquímicas del dolor, entre las que resaltan el dolor óseo, sinovial, así como los factores bioquímicos relacionados con ese síntoma. Se hace referencia a las principales estructuras anatómicas responsables del dolor y sus mecanismos de acción. Se mencionan la relación existente entre ese síntoma y las modalidades imagenológicas, así como los patrones del dolor. Para finalizar, se hace referencia a las escalas de dolor usadas.

Pain is the main symptom of osteoarthritis. Determining the distinctive features of pain in knee osteoarthritis allows for an accurate diagnosis. This article gives a review of the results from research work on the typical features of knee osteoarthritic pain. The mechanics and biochemical causes of pain are described including both bone and synovial biochemical symptom-related factors. The relationships between knee pain, various imaging techniques and pain mechanism are also identified. Finally, the used pain scales are presented.

\section{Introducción}

La artrosis es una entidad que afecta a millones de personas en el mundo. Las articulaciones más afectadas son la cadera, rodilla y columna. Aunque el cartílago articular es un tejido aneural, en la articulación existen estructuras con terminaciones nerviosas que son responsables del dolor. ${ }^{1-3}$

El síntoma fundamental de la artrosis es el dolor, por eso, el $20 \%$ del reporte de ese síntoma a nivel internacional, se debe a esa afección La incidencia aumenta con la edad y obesidad, además de constituir la principal causa de búsqueda de asistencia médica y viene acompañada por lo general, de una sensación de inestabilidad, calor y limitación del movimiento articular. ${ }^{4-6}$

La articulación de la rodilla es afectada en un gran número de enfermos, según la Academia Americana de Cirujanos Ortopédicos, la incidencia de artrosis de la rodilla es de 240 pacientes por cada 100000 habitantes en los Estados Unidos received

September 13, 2018

accepted

September 9, 2019
DOI https://doi.org/

10.1055/s-0039-1698796. ISSN $0716-4548$.
Copyright (e) 2019 by Thieme Revinter

Publicações Ltda, Rio de Janeiro, Brazil
License terms

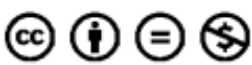


de América. ${ }^{7}$ El dolor es de localización anterior o anteromedial, aparece después de una estadía de pie prolongada o caminata y por lo general se irradia hacia la pierna y es de tipo mecánico. Por otra parte, el paciente refiere ese síntoma como insidioso, variable, intermitente con días de mayor y menor intensidad, puede presentarse al reposo y en horas de la noche, lo que lleva a pensar en un proceso degenerativo avanzado. ${ }^{8-10}$

Según Gwilym SE y col., ${ }^{11}$ el dolor en pacientes con artrosis tiene controversias como: primero, no todos los pacientes con artrosis tienen dolor y no se puede predecir quién de ellos lo tendrá; segundo, ese síntoma tiende a mejorar con técnicas de placebo y, tercero, uno de cada diez pacientes a los que se les realizó una artroplastia total de rodilla continúa con dolor. ${ }^{12,13}$

Los factores asociados al dolor en la artrosis son: sexo femenino, ya que ese sexo refiere más dolor que el hombre en número e intensidad; articulación afectada, la rodilla es más dolorosa que la mano; y presencia de cambios radiográficos severos.

La comprensión del dolor en pacientes con artrosis permite enfocar de manera correcta las decisiones diagnósticas y terapéuticas.

\section{Características Generales del Dolor en la Artrosis de Rodilla}

Las características clínicas del dolor debido a artrosis de la rodilla son descritas por el enfermo como de tipo profundo, sordo, localizado, agravado con la actividad física en especial con carga de peso y se alivia con el reposo. Aunque al principio es intermitente luego con la progresión de la enfermedad degenerativa pasa a ser constante asociado a pérdida de la capacidad funcional del enfermo. ${ }^{14,15}$

El dolor del paciente con artrosis es causado por estímulos mecánicos y bioquímicos. Dentro de los mecánicos podemos encontrar de origen óseo, sinovial, muscular y otros tejidos de localización periférica. ${ }^{14,15}$

Las causas de dolor óseo se deben a periostitis asociadas a la formación de osteofitos y fracturas subcondrales, como consecuencia de la disminución de la irrigación sanguínea y el aumento de la presión intra-ósea, además de la reducción del drenaje venoso del hueso. ${ }^{16-18}$

Por otra parte, las causas de dolor sinovial ocurren como consecuencia de la irritación de las terminaciones nerviosas sensoriales dentro de la capa sinovial, que cubre los osteofitos y la propia inflamación del tejido sinovial por prostaglandinas, leucotrienos y citocinas, que estimulan los nociceptores, además de la distención articular y el edema. ${ }^{19-21}$

El dolor de la artrosis de rodilla también es de origen muscular, a través de la inestabilidad articular típica de esos enfermos, aumenta el estrés sobre la articulación y por ende la activación de los nociceptores.

La afección de los tejidos de localización periférica, juega un papel importante en la fisiopatología del dolor. En pacientes con artrosis las enzimas metaloproteinasas rompen el cartílago articular y producen cambios en la consistencia de su matriz, asociado a eso ocurre aumento de la actividad del condrocito y el desarrollo de osteofitos. El edema que ocurre a nivel del tejido

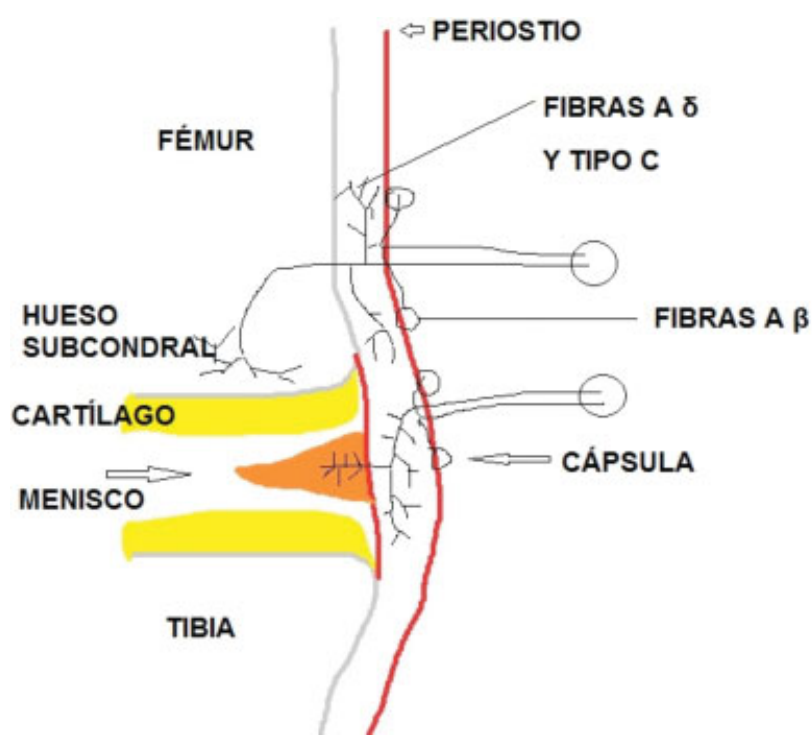

Fig. 1 Estructuras anatómicas responsables del dolor en la rodilla. Fuente: Autores.

sinovial y el aumento de volumen del líquido intersticial dentro de la articulación, aumentan de manera significativa la presión intra y extrarticular. La alteración de todos esos tejidos, activa el sistema nociceptivo que genera dolor. ${ }^{22-24}$

Debido a ese estímulo, se vierten dentro de la articulación mediadores de tipo inflamatorio como: bradicinina, histamina, prostaglandinas, ácido láctico, sustancia $\mathrm{P}$ y péptido $\mathrm{C}$, que desde el punto de vista genético está relacionado con la calcitonina. Todos esos mediadores, disminuyen el umbral doloroso de los tejidos dentro de la articulación y hacen que los mismos sean más sensibles a la respuesta dolorosa. Por otra parte, su liberación, estimula la liberación de más mediadores. ${ }^{25-27}$

La activación de los nociceptores genera señales a través de las fibras mielinizadas $A-\delta y / o$ las fibras no mielinizadas $C$, las que se dirigen a la región dorsal de la médula espinal. Las fibras $A-\delta$ transmiten el dolor agudo y las fibras $C$ el difuso (-Fig. 1). ${ }^{28-30}$

\section{Orígenes del Dolor y su Relación con las Estructuras Anatómicas}

El origen del dolor en pacientes con artrosis puede ser: del hueso subcondral, osteofitos, ligamentos, entesis, cápsula, músculos paravertebrales y tejido sinovial, a través de varios mecanismos como: hipertensión medular, inflamación, distención, entre otros (- Tabla 1). ${ }^{31-33}$

El dolor localizado sirve de referencia de la posible estructura responsable, cuando existe afección de la zona anterior de la rodilla puede ser debido a artrosis patelofemoral, plica sinovial e hipertrofia sinovial anterior. La zona posterior se afecta en caso de lesiones de menisco en sus cuernos posteriores, lesiones de cartílago y quistes poplíteos, como es el caso del descrito por Baker. Las localizaciones mediales y laterales se relacionan con afecciones de meniscos asociadas o no al estrechamiento femorotibial. ${ }^{31,32}$ 
Tabla 1 Origen del dolor en pacientes con artrosis

\begin{tabular}{|l|l|}
\hline Tejido & Mecanismo del dolor \\
\hline Hueso subcondral & $\begin{array}{l}\text { Hipertensión medular y/o } \\
\text { microfractura. }\end{array}$ \\
\hline Osteofitos & $\begin{array}{l}\text { Estiramiento de las terminaciones } \\
\text { nerviosas del periostio. }\end{array}$ \\
\hline Ligamentos & Estiramiento \\
\hline Entesis & Inflamación \\
\hline Cápsula & Inflamación, distención (figura 2) \\
\hline $\begin{array}{l}\text { Músculos } \\
\text { periarticulares }\end{array}$ & Contractura \\
\hline Tejido sinovial & Inflamación \\
\hline
\end{tabular}

Es importante durante la exploración física del paciente en la rodilla, lograr detectar la zona del dolor mediante maniobras, palpación y movimientos pasivos o activos. Un ejemplo típico es el dolor patelofemoral, que es provocado al presionar la rótula sobre los cóndilos femorales. ${ }^{32,33}$

Existe poca correlación entre el dolor y los cambios radiográficos en pacientes con artrosis, pueden detectarse pacientes con dolor de gran intensidad sin o con cambios mínimos desde el punto de vista radiográfico. Por otra parte, hay pacientes con cambios radiográficos marcados y poco o ningún dolor. De allí que la evaluación del compromiso articular es más clínico que imagenológico. ${ }^{34,35}$

\section{Razones que Explican la Disociación entre Parámetros Clínicos e Imagenológicos en la Artrosis}

En el año 1952 Kellgram JH y Lawrence JS citado por Gwilym SE y col., ${ }^{11}$ cuantificaron la relación entre cambios radiográficos y dolor, donde encontraron que solo el $24 \%$ de los pacientes con esos hallazgos radiográficos presentaron dolor. Por otra parte Salaffi F y col., ${ }^{36}$ plantean que el dolor en la artrosis se presenta en un $10 \%$ en enfermos sin cambios radiográficos y de un $40 \%$ a $79 \%$ en los que sí presentan signos positivos de la enfermedad mediante este examen imagenológico.

Las razones para esa pérdida de relación entre el dolor y los exámenes imagenológicos, se debe a varios factores como: la mayoría de las investigaciones radiográficas realizadas son con el paciente en decúbito supino o en carga de peso sin contemplar la articulación patelofemoral y aproximadamente un $24 \%$ de las mujeres afectadas por la artrosis de rodilla, tienen participación de esa articulación; segundo: la radiografía en ocasiones es normal, sin embargo, en el examen artroscópico de la articulación, existen evidencias claras de la presencia de la enfermedad, ya que la radiografía no muestra las alteraciones de la cápsula, cartílago, ligamentos y sinovial; por último, no todo dolor en la rodilla es debido a artrosis, puede ser también debido a bursitis en las inserciones tendinosas de la rodilla $\mathrm{y}$ afecciones de la cadera y columna. ${ }^{36-38}$

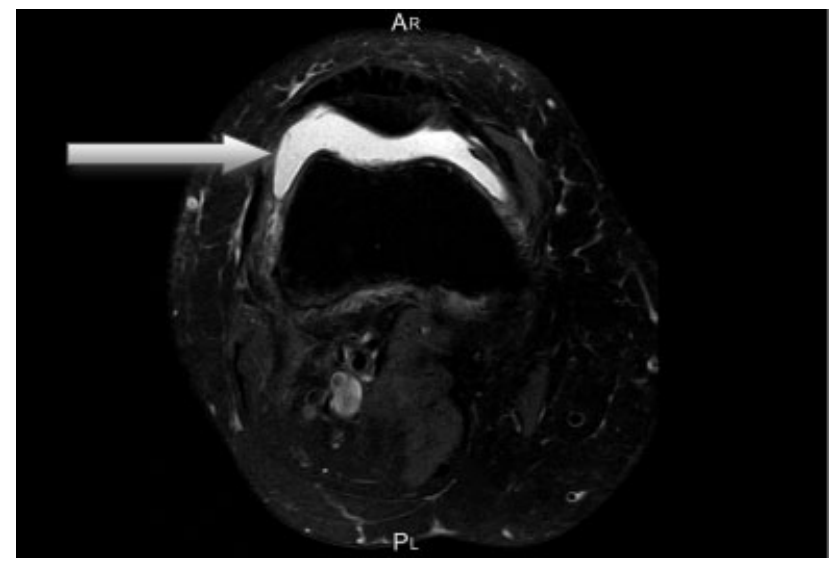

Fig. 2 Vista axial de imagen de resonancia magnética de rodilla derecha, donde se observa líquido articular en el compartimento patelofemoral (flecha de color blanco), lo que provoca distención de la cápsula articular y dolor. Fuente: Autores

\section{Medios Diagnósticos y Patrones del Dolor}

Según Li Q y col., ${ }^{39}$ el ultrasonido de alta definición y la imagen de resonancia magnética de la rodilla, ayudan a identificar las causas de dolor dentro de la articulación, como la distención articular provocada por la cantidad excesiva de líquido sinovial, daño estructural del menisco y cartílago (-Fig. 2). ${ }^{39,40}$

Los patrones de dolor en la artrosis de rodilla son dos, el primero es de tipo generalizado en la zona anterior de la rodilla, y el segundo localizado en la zona inferomedial.Esos patrones son más reconocidos desde el punto de vista clínico que radiográfico. La presencia de osteofitos en la misma localización de los síntomas es un factor de buen pronóstico. $^{41-44}$

El dolor nocturno aunque inusual, es otro patrón reconocido de la artrosis de rodilla, traduce afección severa de la articulación, la intensidad es mayor de 30 según la escala numérica del dolor de 0 a 100 . Por otra parte, un 14,7\% de los enfermos con artrosis refieren que el dolor es más intenso en horas de la noche y un 27,9\% refiere que empeoran con el reposo en cama. Además, la presencia de ese síntoma en horas de la noche, se asocia a la pérdida progresiva de la calidad de vida y se relaciona directamente con la fatiga. ${ }^{45-47}$

\section{Escalas Relacionadas con el Parámetro Dolor}

Debido a que el dolor es el principal síntoma en pacientes con artrosis de la rodilla, la mayoría de los instrumentos evaluativos lo tienen en cuenta dentro de sus parámetros como: la escala Western Ontario and McMaster Universities Osteoarthritis Index (WOMAC), el índice de Lesquene, Knee Injury and Osteoarthritis Outcome Score (KOOS), cuestionario SF-36; la escala visual y numérica al dolor, los cuestionarios Osteoarthritis Research Society International (OARSI), Outcome Measures in Rheumatolgy OMERACT, Intermittent and Constant Osteoarthritis Pain (ICOAP), Osteo-Arthritis Symptom Inventory Scale (OASIS). ${ }^{48-50}$ 


\section{Discusión}

La interpretación del dolor en pacientes con artrosis en especial de la rodilla, es importante para identificar la localización y posible magnitud de la lesión. La minuciosa valoración preoperatoria permite establecer patrones a lo largo del tratamiento, tanto conservador como quirúrgico.

El dolor en pacientes con artrosis de la rodilla, se puede mantener con intensidad variable hasta en un $25 \%$ de los enfermos, a pesar de las diferentes modalidades de tratamiento empleada. ${ }^{7,11}$

El diagnóstico diferencial de pacientes con dolor en la artrosis de rodilla está basado en dos aspectos fundamentales, el primero en lo concerniente al del tipo referido aquí, en este caso como ejemplo: presencia de dolor de la rodilla con origen en la cadera. En el segundo grupo, encontramos entidades locales, por ejemplo: algunos tipos de artritis, tumores óseos, osteomielitis, enfermedades metabólicas, entensopatías, bursitis, inestabilidad por deficiencia de ligamentos y fracturas patológicas, algunas de las cuales pueden concomitar con la artrosis y deben ser sospechadas en caso de respuesta limitada o nula al tratamiento.

El enfrentamiento terapéutico para el alivio de dolor por artrosis de la rodilla está sustentado en tres líneas. La primera está fundamentada en aspectos como: educación del enfermo, reducción del peso corporal, ejercicios físicos, cambios en el estilo de vida, uso de analgésicos simples y medicamentos de aplicación local (-Fig. 3). En relación a la segunda línea de conducta podemos encontrar una gran variedad de modalidades conservadoras como: la indicación de antiinflamatorios no esteroideos y esteroideos, esos últimos tanto por vía sistémica como local, terapia física y de rehabilitación, uso de ortesis, entre otros. La tercera línea está basada, en el tratamiento quirúrgico que incluye: artroscopia, osteotomía y artroplastia tanto parciales como totales. La severidad de la enfermedad se incrementa desde la línea uno a la tres y el número de paciente es mayor en la línea uno que en la tres. Con relación a la segunda línea el tiempo de espera para evaluar la respuesta debe ser al menos de seis semanas. ${ }^{51,52}$

Las escalas descritas para la gradación del dolor son múltiples, pero confirman que ese síntoma es el más importante de todos, y que además facilita evaluar los métodos de tratamiento empleados en esos enfermos.
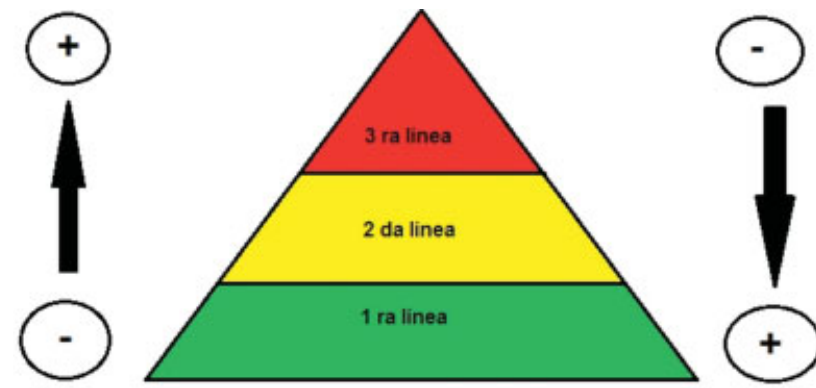

Fig. 3 Líneas de tratamiento para el dolor en la artrosis, señales de la izquierda grado de severidad de la enfermedad, señales de la derecha número de pacientes afectados. Fuente: Autores.
Un aspecto medular, es el efecto placebo en relación al dolor en la artrosis de rodilla, que es importante en dos aspectos, el primero, relacionado con la comparación de medicamentos y procedimientos, ya que permite identificar si tienen mayor y mejor acción sobre el dolor que el placebo, en ese caso tenemos como ejemplo, la administración de medicamentos como el acetaminofén y antiinflamatorios no esteroideos, además, permite evaluar las reacciones adversas. El segundo aspecto de interés, es que existen enfermos que se alivian mediante técnicas de placebo, elemento que puede ser explicado mediante la liberación de endomorfinas. ${ }^{53,54}$

\section{Conclusiones}

El dolor es el principal síntoma en pacientes con artrosis de la rodilla y la principal razón en la búsqueda de asistencia médica. La comprensión adecuada de la fisiopatología y características propias del dolor en la artrosis de rodilla, permite identificar el grado de severidad independientemente de las alteraciones radiográficas asociadas.

\section{Declaración y Verificación de la Presentación}

Este trabajo no ha sido previamente publicado ni presentado simultáneamente en otra revista y tiene la autorización de los autores y sus establecimientos asistenciales.

\section{Conflicto de Intereses}

Lo autor del trabajo declara no tener ningún conflicto de intereses.

\section{Bibliografía}

1 Abdel-Aziem AA, Soliman ES, Mosaad DM, Draz AH. Effect of a physiotherapy rehabilitation program on knee osteoarthritis in patients with different pain intensities. J Phys Ther Sci 2018;30 (02):307-312

2 Cottam WJ, Iwabuchi SJ, Drabek MM, Reckziegel D, Auer DP. Altered connectivity of the right anterior insula drives the pain connectome changes in chronic knee osteoarthritis. Pain 2018; 159(05):929-938

3 Ebell MH. Osteoarthritis: rapid evidence review. Am Fam Physician 2018;97(08):523-526

4 Walton DM, Elliott JM. A new clinical model for facilitating the development of pattern recognition skills in clinical pain assessment. Musculoskelet Sci Pract 2018;36(36):17-24

5 Tran J, Peng PWH, Lam K, Baig E, Agur AMR, Gofeld M. Anatomical study of the innervation of anterior knee joint capsule: implication for image-guided intervention. Reg Anesth Pain Med 2018;43(04):407-414

6 Taylor N. Nonsurgical management of osteoarthritis knee pain in the older adult. Clin Geriatr Med 2017;33(01):41-51

7 Treatment of osteoarthritis of the knee. Evidence based guideline. 2 nd ed. American Academy of Orthopaedic Surgeons. 2013:8-10.

8 Eberly L, Richter D, Comerci G, et al. Psychosocial and demographic factors influencing pain scores of patients with knee osteoarthritis. PLoS One 2018;13(04):e0195075

9 Holden MA, Whittle R, Healey EL, et al. Content and evaluation of the benefits of effective exercise for older adults with knee pain trial physiotherapist training program. Arch Phys Med Rehabil 2017;98(05):866-873 
10 Kim GJ, Choi J, Lee S, Jeon C, Lee K. The effects of high intensity laser therapy on pain and function in patients with knee osteoarthritis. J Phys Ther Sci 2016;28(11):3197-3199

11 Gwilym SE, Pollard TCB, Carr AJ. Understanding pain in osteoarthritis. J Bone Joint Surg Br 2008;90(03):280-287

12 Harden RN, Saracoglu M, Connolly S, et al. "Managing” the placebo effect: the single-blind placebo lead-in response in two pain models. Pain Med 2016;17(12):2305-2310

13 Vaishya R, Pandit R, Agarwal AK, Vijay V. Intra-articular hyaluronic acid is superior to steroids in knee osteoarthritis: A comparative, randomized study. J Clin Orthop Trauma 2017;8 (01):85-88

14 Bobić Lucić L, Grazio S. Impact of balance confidence on daily living activities of older people with knee osteoarthritis with regard to balance, physical function, pain, and quality of life: a preliminary report. Clin Gerontol 2018;41(04):357-365

15 Thakral M, Shi L, Foust JB, et al. Pain quality descriptors in community-dwelling older adults with nonmalignant pain. Pain 2016;157(12):2834-2842

16 Garg Y, Singh J, Sohal HS, Gore R, Kumar A. Comparison of clinical effectiveness and safety of newer nonsteroidal anti-inflammatory drugs in patients of osteoarthritis of knee joint: a randomized, prospective, open-label parallel-group study. Indian J Pharmacol 2017;49(05):383-389

17 Luz-Santos C, Ribeiro Camatti J, Barbosa Paixão A, et al. Additive effect of tDCS combined with Peripheral Electrical Stimulation to an exercise program in pain control in knee osteoarthritis: study protocol for a randomized controlled trial. Trials 2017;18(01):609

18 Salamh P, Cook C, Reiman MP, Sheets C. Treatment effectiveness and fidelity of manual therapy to the knee: A systematic review and meta-analysis. Musculoskelet Care 2017;15(03):238-248

19 Conaghan PG, Hunter DJ, Cohen SB, et al; FX006-2014-008 Participating Investigators. Effects of a single intra-articular injection of a microsphere formulation of triamcinolone acetonide on knee osteoarthritis pain: a double-blinded, randomized, placebo-controlled, multinational study. J Bone Joint Surg Am 2018;100(08):666-677

20 da Costa BR, Hari R, Jüni P. Intra-articular corticosteroids for osteoarthritis of the knee. JAMA 2016;316(24):2671-2672

21 Ismail A, Moore C, Alshishani N, Yaseen K, Alshehri MA. Cognitive behavioural therapy and pain coping skills training for osteoarthritis knee pain management: a systematic review. J Phys Ther Sci 2017;29(12):2228-2235

22 Yoon J, Kanamori A, Fujii K, Isoda H, Okura T. Evaluation of maslinic acid with whole-body vibration training in elderly women with knee osteoarthritis. PLoS One 2018;13(03):e0194572

23 Laires PA, Laíns J, Miranda LC, et al. Inadequate pain relief among patients with primary knee osteoarthritis. Rev Bras Reumatol Engl Ed 2017;57(03):229-237

24 MacFarlane LA, Yang H, Collins JE, et al. Associations among meniscal damage, meniscal symptoms and knee pain severity. Osteoarthritis Cartilage 2017;25(06):850-857

25 Zacaron KAM, da Silva Mendes N, E Silva YC, Lucchetti G. Effects of laying on of hands with and without a spiritual context on pain and functionality of older women with knee osteoarthritis: study protocol for a randomized controlled trial. J Integr Med 2018;16 (02):106-112

26 Gutiérrez-Gómez JJ, Santillán-Barrera E, Velasco-Legoff C, ChávezEspina LM, Torres-Roldán F, Aldrete-Velasco J. [Multidisciplinary meeting on application techniques for restitution of synovial fluid in patients with knee arthritis: update]. Acta Ortop Mex 2017;31 (06):319-327

27 Kuntz AB, Chopp-Hurley JN, Brenneman EC, et al. Efficacy of a biomechanically-based yoga exercise program in knee osteoarthritis: A randomized controlled trial. PLoS One 2018;13 (04):e0195653

28 Wang Y, Lombard C, Hussain SM, et al. Effect of a low-intensity, selfmanagement lifestyle intervention on knee pain in community- based young to middle-aged rural women: a cluster randomised controlled trial. Arthritis Res Ther 2018;20(01):74

29 Dimitroulas T, Lambe T, Klocke R, Kitas GD, Duarte RV. Biologic drugs as analgesics for the management of osteoarthritis. Semin Arthritis Rheum 2017;46(06):687-691

30 Hamdi A, Albaghdadi AT, Ghalimah B, Alnowiser A, Ahmad A, Altaf A. Bariatric surgery improves knee function and not knee pain in the early postoperative period. J Orthop Surg Res 2018;13(01):82

31 Hagen KB, Grotle M. Critically Appraised Papers: Moderateintensity walking for people with severe knee osteoarthritis does not decrease pain but may have cardiovascular benefits [synopsis]. J Physiother 2018;64(02):123

32 Kim MK, Ha CW, In Y, et al. A multicenter, double-blind, phase III clinical trial to evaluate the efficacy and safety of a cell and gene therapy in knee osteoarthritis patients. Hum Gene Ther Clin Dev 2018;29(01):48-59

33 Owens C, Conaghan PG. Improving joint pain and function in osteoarthritis. Practitioner 2016;260(1799):17-20

34 Eckstein F, Guermazi A, Gold G, et al. Imaging of cartilage and bone: promises and pitfalls in clinical trials of osteoarthritis. Osteoarthritis Cartilage 2014;22(10):1516-1532

35 Bastick AN, Belo JN, Runhaar J, Bierma-Zeinstra SM. What are the prognostic factors for radiographic progresión of knee osteoarthritis: A meta analysis. Clin Orthop Relat Res 2015;473 (09):2969-2989

36 Salaffi F, Ciapetti A, Carotti M. The sources of pain in osteoarthritis: a pathophysiological review. Reumatismo 2014; 66(01):57-71

37 Fernandes GS, Parekh SM, Moses J, et al. Prevalence of knee pain, radiographic osteoarthritis and arthroplasty in retired professional footballers compared with men in the general population: a crosssectional study. Br J Sports Med 2018;52(10):678-683

38 Sheehy L, Culham E, McLean L, et al. Validity and sensitivity to change of three scales for the radiographic assessment of knee osteoarthritis using images from the Multicenter Osteoarthritis Study (MOST). Osteoarthritis Cartilage 2015;23(09):1491-1498

$39 \mathrm{Li} \mathrm{Q}$ Amano $\mathrm{K}$, Link TM, Ma CB. Advanced imaging in osteoarthritis. Sports Health 2016;8(05):418-428

40 Schwaiger BJ, Gersing AS, Mbapte Wamba J, Nevitt MC, McCulloch CE, Link TM. Can signal abnormalities detected with MR imaging in knee articular cartilage be used to predict development of morphologic cartilage defects? 48 month data from the osteoarthritis initiative. Radiology 2016;281(01):158-167

41 Farpour HR, Fereydooni F. Comparative effectiveness of intraarticular prolotherapy versus peri-articular prolotherapy on pain reduction and improving function in patients with knee osteoarthritis: A randomized clinical trial. Electron Physician 2017;9(11):5663-5669

42 Taheri P, Vahdatpour B, Asl MM, Ramezanian H. Effects of taping on pain and functional outcome of patients with knee osteoarthritis: a pilot randomized single-blind clinical trial. Adv Biomed Res 2017;6(06):139

43 Lee MC, Saleh R, Achuthan A, et al. CCL17 blockade as a therapy for osteoarthritis pain and disease. Arthritis Res Ther 2018;20(01):62

44 Rafanan BS Jr, Valdecañas BF, Lim BP, et al. Consensus recommendations for managing osteoarthritic pain with topical NSAIDs in Asia-Pacific. Pain Manag 2018;8(02):115-128

45 Hart HF, Barton CJ, Khan KM, Riel H, Crossley KM. Is body mass index associated with patellofemoral pain and patellofemoral osteoarthritis? A systematic review and meta-regression and analysis. Br J Sports Med 2017;51(10):781-790

46 Sibille KT, Chen H, Bartley EJ, et al. Accelerated aging in adults with knee osteoarthritis pain: consideration for frequency, intensity, time, and total pain sites. Pain Rep 2017;2(03):e591

47 El-Hakeim EH, Elawamy A, Kamel EZ, et al. Fluoroscopic guided radiofrequency of genicular nerves for pain alleviation in chronic knee osteoarthritis: a single-blind randomized controlled trial. Pain Physician 2018;21(02):169-177 
48 Abdalbary SA. Ultrasound with mineral water or aqua gel to reduce pain and improve the WOMAC of knee osteoarthritis. Future Sci OA 2016;2(01):FSO110

49 Salazar-Guzmán I, Garfias-Rosas J, Butze-Rangel W. [Comparison of the clinical response to the application of Hylan G-F 20 and collagen-PVP in patients with knee osteoarthritis]. Acta Ortop Mex 2017;31(06):283-286

50 Lee M, Yoo J, Kim JG, et al. A randomized, multicenter, phase III trial to evaluate the efficacy and safety of polmacoxib compared with celecoxib and placebo for patients with osteoarthritis. Clin Orthop Surg 2017;9(04):439-457

51 Gregori D, Giacovelli G, Minto C, et al. Association of pharmacological treatments with long-term pain control in patients with knee osteoarthritis: a systematic review and meta-analysis. JAMA 2018;320(24):2564-2579

52 Li JS, Tsai TY, Clancy MM, Li G, Lewis CL, Felson DT. Weight loss changed gait kinematics in individuals with obesity and knee pain. Gait Posture 2019;68:461-465

53 Dworkin RH, Turk DC, Peirce-Sandner S, et al. Meta-analysis of assay sensitivity and study features in clinical trials of pharmacologic treatments for osteoarthritis pain. Arthritis Rheumatol 2014;66(12):3327-3336

54 Gallagher B, Tjoumakaris FP, Harwood MI, Good RP, Ciccotti MG, Freedman KB. Chondroprotection and the prevention of osteoarthritis progression of the knee: a systematic review of treatment agents. Am J Sports Med 2015;43(03):734-744 